\title{
QUASILINEAR ELLIPTIC SYSTEMS OF RESONANT TYPE AND NONLINEAR EIGENVALUE PROBLEMS
}

\author{
PABLO L. DE NÁPOLI AND M. CRISTINA MARIANI
}

Received 20 February 2001

This work is devoted to the study of a quasilinear elliptic system of resonant type. We prove the existence of infinitely many solutions of a related nonlinear eigenvalue problem. Applying an abstract minimax theorem, we obtain a solution of the quasilinear system $-\Delta_{p} u=F_{u}(x, u, v),-\Delta_{q} v=F_{v}(x, u, v)$, under conditions involving the first and the second eigenvalues.

\section{Introduction}

1.1. The problem and some previous results. We consider a gradient elliptic system

$$
-\Delta_{p} u=F_{u}(x, u, v), \quad-\Delta_{q} v=F_{v}(x, u, v)
$$

Elliptic problems involving the $p$-Laplacian have been studied by several authors (cf. $[3,7,8,10,11])$. We recall some results from the work of Boccardo and de Figueiredo [4].

It is well known that the solutions of (1.1) in $W=W_{0}^{1, p}(\Omega) \times W_{0}^{1, q}(\Omega)$ are the critical points of the functional

$$
\Phi(u, v)=\frac{1}{p} \int_{\Omega}|\nabla u|^{p}+\frac{1}{q} \int_{\Omega}|\nabla v|^{q}-\int_{\Omega} F(x, u, v)
$$

under the following three assumptions:

(1) $\Omega \subset \mathbb{R}^{N}$ is a bounded domain, $1<p, q<N$, so that the following continuous embeddings hold:

$$
W_{0}^{1, p}(\Omega) \subset L^{p^{*}}(\Omega), \quad W_{0}^{1, q}(\Omega) \subset L^{q^{*}}(\Omega)
$$


(2) $F: \bar{\Omega} \times \mathbb{R} \times \mathbb{R} \rightarrow \mathbb{R}$ is $C^{1}$ and verifies the following growth condition:

$$
|F(x, s, t)| \leq c\left(1+|s|^{p^{*}}+|t|^{q^{*}}\right) \quad \forall x \in \bar{\Omega} ; s, t \in \mathbb{R} ;
$$

(3) in order to have $\Phi \in C^{1}(W, \mathbb{R})$, we assume

$$
\begin{array}{ll}
\left|F_{s}(x, s, t)\right| \leq C\left(1+|s|^{p^{*}-1}+|t|^{q^{*}\left(p^{*}-1\right) / p^{*}}\right) & \forall x \in \bar{\Omega} ; s, t \in \mathbb{R}, \\
\left|F_{t}(x, s, t)\right| \leq C\left(1+|t|^{q^{*}-1}+|s|^{p^{*}\left(q^{*}-1\right) / q^{*}}\right) & \forall x \in \bar{\Omega} ; s, t \in \mathbb{R} .
\end{array}
$$

The geometry of $\Phi$ depends strongly on the values of $\alpha$ and $\beta$ in the estimate

$$
|F(x, s, t)| \leq c\left(1+|s|^{\alpha}+|t|^{\beta}\right) \quad \forall x \in \bar{\Omega} ; s, t \in \mathbb{R},
$$

where $\alpha \leq p^{*}, \beta \leq q^{*}$. In this work we are interested in the case $\alpha=p, \beta=q$ (systems of resonant type).

In our case, it is quite adequate to assume the following condition on $F$ : consider the function

$$
L(x, s, t)=\frac{1}{p} F_{s}(x, s, t) s+\frac{1}{q} F_{t}(x, s, t) t-F(x, s, t) .
$$

Assume that

$$
\lim _{\|(s, t)\| \rightarrow \infty} L(x, s, t)= \pm \infty \quad \text { uniformly for } x \in \Omega .
$$

This assumption implies that $\Phi$ satisfies the following compactness Cerami condition.

Definition 1.1. Let $X$ be a Banach space and $\Phi \in C^{1}(X, \mathbb{R})$. Given $c \in \mathbb{R}$, we say that $\Phi$ satisfies condition $\left(C_{c}\right)$, if

(1) any bounded sequence $\left(u_{n}\right) \subset X$ such that $\Phi\left(u_{n}\right) \rightarrow c$ and $\Phi^{\prime}\left(u_{n}\right) \rightarrow 0$ has a convergent subsequence;

(2) there exist constants $\delta, R, \alpha>0$ such that

$$
\left\|\Phi^{\prime}(u)\right\|\|u\| \geq \alpha \quad \forall u \in \Phi^{-1}([c-\delta, c+\delta]) \text { with }\|u\| \geq R .
$$

If $\Phi \in C^{1}(X, \mathbb{R})$ satisfies condition $\left(C_{c}\right)$ for every $c \in \mathbb{R}$, we say that $\Phi$ satisfies condition $(C)$.

Condition $(C)$ was introduced by Cerami [5]. It was shown in [2] that from condition $(C)$ it is possible to obtain a deformation lemma, that is fundamental in order to get minimax theorems.

In order to avoid resonance, Boccardo and de Figueiredo [4] introduced an assumption on $F$ involving an eigenvalue problem

$$
\begin{aligned}
-\Delta_{p} u-a G_{u}(u, v) & =\lambda|u|^{p-2} u, \\
-\Delta_{q} v-a G_{v}(u, v) & =\lambda|v|^{q-2} v,
\end{aligned}
$$


where $a=a(x) \in L^{\infty}(\Omega)$ and $G$ is a $C^{1}$ even function $G: \mathbb{R} \rightarrow[0, \infty)$ such that

$$
\begin{gathered}
G\left(c^{1 / p} s, c^{1 / q} t\right)=c G(s, t) \quad \forall c>0, \\
G(s, t) \leq K\left(\frac{1}{p}|s|^{p}+\frac{1}{q}|t|^{q}\right) .
\end{gathered}
$$

We call such a $G$ a $(p, q)$ homogeneous function.

It is easy to see that (1.11) implies (1.12). A $(p, q)$-homogeneous function satisfies

$$
\frac{1}{p} G_{s}(s, t) s+\frac{1}{q} G_{t}(s, t) t=G(s, t) .
$$

Examples of $(p, q)$ homogeneous functions are

(1) $G(s, t)=c_{1}|s|^{p}+c_{2}|t|^{q}$,

(2) $G(s, t)=c|s|^{\alpha}|t|^{\beta}$ with $\alpha / p+\beta / q=1$ where $c, c_{1}, c_{2}$ are constants.

The following results are proved in [4].

Theorem 1.2. Problem (1.10), with $G$ as above, has a first eigenvalue $\lambda_{1}(a)$, characterized variationally by

$$
\lambda_{1}(a)=\inf _{(u, v) \neq(0,0)} \frac{(1 / p) \int_{\Omega}|\nabla u|^{p}+(1 / q) \int_{\Omega}|\nabla v|^{q}-\int_{\Omega} a G(u, v)}{(1 / p) \int_{\Omega}|u|^{p}+(1 / q) \int_{\Omega}|v|^{q}}
$$

which depends continuously on a in the $L^{\infty}$-norm.

Theorem 1.3. Assume (1.5), (1.6) with $\alpha=p, \beta=q$, and that the following conditions hold:

(1) there exist positive numbers $c, R, \mu$, and $v$ such that

$$
\frac{1}{p} s F_{s}(x, s, t)+\frac{1}{q} t F_{t}(x, s, t)-F(x, s, t) \geq c\left(|s|^{\mu}+|t|^{v}\right) \quad \text { for }|s|,|t|>R,
$$

(2) there exists $G$ as above, such that

$$
\lim \sup _{|s|,|t| \rightarrow \infty} \frac{F(x, s, t)}{G(s, t)} \leq a(x) \in L^{\infty}(\Omega),
$$

where $\lambda_{1}(a)>0$.

Then the functional $\Phi$ is bounded from below and the infimum is achieved.

1.2. The existence of infinitely many eigenfunctions. Let $\mathscr{C}$ be the class of compact symmetric $(C=-C)$ subsets of the space $W$. We recall that for $C \in \mathscr{C}$ the Krasnoselskii genus gen $(C)$ is defined as the minimum integer $n$ such that there exists an odd continuous mapping $\varphi: C \rightarrow\left(\mathbb{R}^{n}-\{0\}\right)$ (cf. [1]). We note

$$
\mathscr{C}_{k}=\{C \in \mathscr{C}: \operatorname{gen}(C) \geq k\} \text {. }
$$


For an arbitrary symmetric subset $S$ of $W-\{0\}$ the genus over compact sets $\gamma(S)$ is defined by

$$
\gamma(S)=\sup \{\operatorname{gen}(C): C \subset S, C \in \mathscr{C}, C \text { compact }\} .
$$

Now we may state our main result on the eigenvalue problem.

Theorem 1.4. The eigenvalue problem (1.10), with $G$ as above, has infinitely many eigenfunctions given by

$$
\lambda_{k}(a, G)=\inf _{C \in \mathscr{C}_{k}(u, v) \in C} \sup _{\frac{(1 / p) \int_{\Omega}|\nabla u|^{p}+(1 / q) \int_{\Omega}|\nabla v|^{q}-\int_{\Omega} a G(u, v)}{(1 / p) \int_{\Omega}|u|^{p}+(1 / q) \int_{\Omega}|v|^{q}}}
$$

and $\lambda_{k}(a, G) \rightarrow \infty$ as $k \rightarrow \infty$. Moreover, $\lambda_{k}$ depends continuously on $a$ in the $L^{\infty}$ norm.

Remark 1.5. Equivalently if we define

$$
S=\left\{(u, v) \in W: \frac{1}{p} \int_{\Omega}|u|^{p}+\frac{1}{q} \int_{\Omega}|v|^{q}=1\right\},
$$

we have

$$
\lambda_{k}(a, G)=\inf _{C \in \mathscr{C}_{k}, C \subset S} \sup _{(u, v) \in C} \frac{1}{p} \int_{\Omega}|\nabla u|^{p}+\frac{1}{q} \int_{\Omega}|\nabla v|^{q}-\int_{\Omega} a G(u, v) .
$$

We will write $\lambda_{k}(a)$ instead of $\lambda_{k}(a, G)$, when the dependence on the $(p, q)$ homogeneous function $G$ is clear from the context.

1.3. The existence result for resonant systems. Applying Theorem 1.4 and an abstract minimax principle from [9], we prove the following theorem.

Theorem 1.6. Assume that $F: \Omega \times \mathbb{R} \times \mathbb{R} \rightarrow \mathbb{R}$ verifies (1.5), (1.6) with $\alpha=p$, $\beta=q,(1.8)$, and that $a_{1}, a_{2} \in L^{\infty}(\Omega)$ satisfy

$$
a_{1}(x) \leq \lim \inf _{|s|,|t| \rightarrow \infty} \frac{F(x, s, t)}{G_{1}(s, t)} \leq \lim \sup _{|s|,|t| \rightarrow \infty} \frac{F(x, s, t)}{G_{2}(s, t)} \leq a_{2}(x)
$$

with $G_{1}$ and $G_{2}$ two $(p, q)$-homogeneous functions and $\lambda_{1}\left(a_{1}, G_{1}\right)<0<\lambda_{2}\left(a_{2}, G_{2}\right)$, where $\lambda_{1}\left(a_{1}, G_{1}\right), \lambda_{2}\left(a_{2}, G_{2}\right)$ are given by (1.19). Then problem (1.1) has at least one solution.

Remark 1.7. The conditions above could be reformulated in terms of a different eigenvalue problem, for $a \in L^{\infty}(\Omega), a(x)>0$

$$
-\Delta_{p} u=\mu a G_{u}(u, v), \quad-\Delta_{q} v=\mu a G_{v}(u, v) .
$$

This problem also has infinitely many eigenvalues given by

$$
\mu_{k}(a)=\inf _{C \in \mathscr{C}_{k}} \sup _{(u, v) \in C} \frac{(1 / p) \int_{\Omega}|\nabla u|^{p}+(1 / q) \int_{\Omega}|\nabla v|^{p}}{\int_{\Omega} a G(u, v)} .
$$


The condition $\lambda_{1}(a)<0$ is equivalent to $\mu_{1}(a)<1$, and the condition $\lambda_{2}(a)>0$ is equivalent to $\mu_{2}(a)>1$.

Remark 1.8. As an example for Theorem 1.6, we may take

$$
G_{1}(s, t)=G_{2}(s, t)=|s|^{\alpha}|t|^{\beta}
$$

with $\alpha / p+\beta / q=1$;

$$
F(x, s, t)=\lambda|s|^{\alpha}|t|^{\beta}+c|s|^{\mu}|t|^{\delta},
$$

where $c \neq 0$ is a constant, and we assume that

$$
\mu_{1}(1)<\lambda<\mu_{2}(1)
$$

and $\mu<\alpha, \delta<\beta$, where $\mu_{1}(1), \mu_{2}(1)$ are defined as above (with $a \equiv 1$ ).

\section{The eigenvalue problem}

2.1. The functional framework. We apply the following abstract theorem due to Amann [1].

THeorem 2.1. Suppose that the following hypotheses are satisfied:

- $X$ is a real Banach space of infinite dimension, that is uniformly convex;

- $A: X \rightarrow X^{*}$ is an odd potential operator (i.e., $A$ is the Gateaux derivative of $A: X \rightarrow \mathbb{R})$ which is uniformly continuous on bounded sets, and satisfies condition $(S)_{1}$ : if $u_{j} \rightarrow u$ (weakly in $X$ ) and $A\left(u_{j}\right) \rightarrow v$, then $u_{j} \rightarrow u$ (strongly in $X$ ).

- For a given constant $\alpha>0$, the level set

$$
M_{\alpha}=\{u \in X: \mathscr{A}(u)=\alpha\}
$$

is bounded and each ray through the origin intersects $M_{\alpha}$. Moreover, for every $u \neq 0,\langle A(u), u\rangle>0$ and there exists a constant $\rho_{\alpha}>0$ such that $\langle A(u), u\rangle \geq \rho_{\alpha}$ on $M_{\alpha}$.

- The mapping $B: X \rightarrow X^{*}$ is a strongly sequentially continuous odd potential operator (with potential $\mathscr{B}$ ), such that $\mathscr{B}(u) \neq 0$ implies that $B(u) \neq 0$. Let

$$
\beta_{k}=\sup _{C \in \mathscr{C}, C \subset M_{\alpha}} \inf _{u \in C} \mathscr{B}(u)
$$

Then if $\beta_{k}>0$, there exists an eigenfunction $u_{k} \in M_{\alpha}$ with $\mathscr{B}(u)=\beta_{k}$. If

$$
\gamma\left(\left\{u \in M_{\alpha}: \mathscr{B}(u) \neq 0\right\}\right)=\infty,
$$

then there exist infinitely many eigenfunctions. 
We will work in the Banach space

$$
W=W_{0}^{1, p}(\Omega) \times W_{0}^{1, q}(\Omega)
$$

equipped with the norm

$$
\|(u, v)\|_{W}=\sqrt{\|u\|_{p}^{2}+\|v\|_{q}^{2}} .
$$

As each factor is uniformly convex, we can conclude that $W$ is uniformly convex (see [6]). Given $\left(u^{*}, v^{*}\right) \in W^{-1, p^{\prime}}(\Omega) \oplus W^{-1, q^{\prime}}(\Omega)$ we may think of it as an element of $W^{*}$ :

$$
\left\langle\left(u^{*}, v^{*}\right),(u, v)\right\rangle=\left\langle u^{*}, u\right\rangle+\left\langle v^{*}, v\right\rangle .
$$

Then we have $W^{*} \cong W^{-1, p^{\prime}}(\Omega) \oplus W^{-1, q^{\prime}}(\Omega)$ (isometric isomorphism), where the norm in $W^{*}$ is given by

$$
\left\|\left(u^{*}, v^{*}\right)\right\|_{W^{*}}=\sqrt{\left\|u^{*}\right\|^{2}+\left\|v^{*}\right\|^{2}} .
$$

With the notations of Theorem 2.1, we define

$$
\begin{aligned}
& \mathscr{A}_{0}(u, v)=\frac{1}{p} \int_{\Omega}|\nabla u|^{p}+\frac{1}{q} \int_{\Omega}|\nabla v|^{q}, \\
& \mathscr{A}(u, v)=\mathscr{A}_{0}(u, v)-\int_{\Omega} a G(u, v)+M\left(\frac{1}{p} \int_{\Omega}|u|^{p}+\frac{1}{q} \int_{\Omega}|v|^{q}\right),
\end{aligned}
$$

with $a$ and $G$ as in the statement of Theorem 1.4, and $M$ a fixed constant such that $M>K\|a\|_{L^{\infty}}$, where $K$ is the constant in (1.12).

We write $\mathscr{A}_{a}$ instead of $\mathscr{A}$ when we want to remark the dependence on the weight $a$

$$
\begin{aligned}
A(u, v) & =\left(-\Delta_{p} u-a G_{u}(u, v)+M|u|^{p-2} u,-\Delta_{q} v-a G_{q}(u, v)+M|v|^{q-2} v\right), \\
\mathscr{B}(u, v) & =\frac{1}{p} \int_{\Omega}|u|^{p}+\frac{1}{q} \int_{\Omega}|v|^{q}, \\
B(u, v) & =\left(|u|^{p-2} u,|v|^{q-2} v\right) .
\end{aligned}
$$

In order to apply Theorem 2.1, we prove the following two lemmas.

LEMMA 2.2. (1) A is uniformly continuous on bounded sets.

(2) A verifies the $(S)_{1}$ condition.

Proof. We write $A=A_{1}-A_{2}$, where

$$
\begin{aligned}
& A_{1}(u, v)=\left(-\Delta_{p} u,-\Delta_{q} v\right), \\
& A_{2}(u, v)=\left(a G_{u}(u, v)-M|u|^{p-2} u, a G_{v}(u, v)-M|v|^{q-2} v\right) .
\end{aligned}
$$


We claim that $A_{2}: W \rightarrow W^{*}$ verifies that: if $\left(u_{j}, v_{j}\right) \rightarrow(u, v)$ in $W$, then $A_{2}\left(u_{j}, v_{j}\right)$ $\rightarrow A_{2}(u, v)$ in $W^{*}$.

Indeed, if $\left(u_{j}, v_{j}\right) \rightarrow(u, v)$, then

$$
\left(u_{j}, v_{j}\right) \longrightarrow(u, v) \quad \text { in } L^{p}(\Omega) \times L^{q}(\Omega)
$$

and we obtain that

$$
\begin{array}{ll}
G_{u}\left(u_{j}, v_{j}\right) \longrightarrow G_{u}(u, v) & \text { in } L^{p^{\prime}}(\Omega), \\
G_{v}\left(u_{j}, v_{j}\right) \longrightarrow G_{v}(u, v) & \text { in } L^{q^{\prime}}(\Omega) .
\end{array}
$$

Hence, $A_{2}\left(u_{j}, v_{j}\right) \rightarrow A_{2}(u, v)$ in $W^{*}$.

Let $\left(u_{j}, v_{j}\right) \rightarrow(u, v)$ in $W$ such that

$$
A\left(u_{j}, v_{j}\right) \longrightarrow(z, w)
$$

Therefore $A_{2}\left(u_{j}, v_{j}\right) \rightarrow A_{2}(u, v)$ and then $A_{1}\left(u_{j}, v_{j}\right) \rightarrow(z, w)+A_{2}(u, v)$. Since $A_{1}$ verifies condition $(S)_{1}$, it follows that $\left(u_{j}, v_{j}\right) \rightarrow(u, v)$.

Lemma 2.3. (1) The set $M_{\alpha}=\{(u, v) \in W: \mathscr{A}=\alpha\}$ is bounded.

(2) Every ray $t \cdot(u, v)$ with $(u, v) \neq 0$ intersects $M_{\alpha}$.

(3) There exists a constant $\rho_{\alpha}>0$ such that

$$
\langle A(u, v),(u, v)\rangle \leq \rho_{\alpha} .
$$

(4) Condition (2.3) is satisfied.

Proof. (1) As we have fixed $M>K\|a\|_{L^{\infty}}$ on $M_{\alpha}$, then

$$
\alpha=\mathscr{A}(u, v) \geq \frac{1}{p}|\nabla u|^{p}+\frac{1}{q}|\nabla v|^{q}
$$

and the proof is complete.

(2) Let $f(c)=\mathscr{A}(c(u, v)), f(0)=0$,

$$
\begin{aligned}
f(c)= & \frac{c^{p}}{p} \int_{\Omega}|\nabla u|^{p}+\frac{c^{q}}{q} \int_{\Omega}|\nabla v|^{q} \\
& -\int_{\Omega} a G(c u, c v)+M\left(\frac{c^{p}}{p} \int_{\Omega}|u|^{p}+\frac{c^{q}}{q} \int_{\Omega}|v|^{q}\right) .
\end{aligned}
$$

From (1.12) and the choice of $M$, we have

$$
f(c) \geq \frac{c^{p}}{p} \int_{\Omega}|\nabla u|^{p}+\frac{c^{q}}{q} \int_{\Omega}|\nabla v|^{q} \longrightarrow+\infty
$$

as $c \rightarrow \infty$. Since $f$ is continuous, there exists $c \in \mathbb{R}$ such that $f(c)=\alpha$. 
(3) We have

$$
\begin{aligned}
\langle A(u, v),(u, v)\rangle= & \int_{\Omega}|\nabla u|^{p}+\int_{\Omega}|\nabla v|^{q} \\
& -\int_{\Omega} a\left[G_{u}(u, v) u+G_{v}(u, v) v\right]+M\left(\int_{\Omega}|u|^{p}+\int_{\Omega}|v|^{q}\right) .
\end{aligned}
$$

Then, using (1.13)

$$
\langle A(u, v),(u, v)\rangle \geq \min \{p, q\} \mathscr{A}(u, v)=\min \{p, q\} \alpha .
$$

(4) In order to see that $\gamma\left(M_{\alpha}\right) \geq k$, it is enough to show that $M_{\alpha}$ contains subsets homeomorphic to the unit sphere in $\mathbb{R}^{k}$ by an odd homeomorphism. Hence, the proof is completed.

2.2. The continuous dependence of $\lambda_{k}(a)$ on $a$. In this section we prove that the eigenvalue $\lambda_{k}(a)$ depends continuously on the weight $a$ in the $L^{\infty}$-norm. This result will be used for proving Lemma 3.3.

Proposition 2.4. The eigenvalue $\lambda_{k}(a)$ depends continuously on $a$ in the $L^{\infty}$ norm.

Proof. We have

$$
\left|\mathscr{A}_{a}(u, v)-\mathscr{A}_{b}(u, v)\right| \leq K\|a-b\|_{L^{\infty}}\left(\frac{1}{p} \int_{\Omega}|u|^{p}+\frac{1}{q} \int_{\Omega}|v|^{q}\right),
$$

where $K$ is given by condition (1.12), with $\mathscr{A}_{a}, \mathscr{A}_{b}$ as above. Let $\varepsilon>0$. Then there exists $C \in \mathscr{C}_{k}, C \subset S$ such that

$$
\sup _{(u, v) \in C} \mathscr{A}_{a}(u, v) \leq \lambda_{k}(a)+\frac{\varepsilon}{2} .
$$

Then for any $(u, v) \in C$, if $\|a-b\|_{L^{\infty}} \leq \delta=\varepsilon / 2 K$ we get

$$
\mathscr{A}_{b}(u, v) \leq \mathscr{A}_{a}(u, b)+\frac{\varepsilon}{2} \leq \lambda_{k}(a)+\varepsilon .
$$

It follows that

$$
\sup _{(u, v) \in C} \mathscr{A}_{b}(u, v) \leq \lambda_{2}(a)+\varepsilon
$$

and we obtain

$$
\lambda_{k}(b) \leq \lambda_{k}(a)+\varepsilon
$$

By reversing the roles of $a$ and $b$, we get $\left|\lambda_{k}(a)-\lambda_{k}(b)\right| \leq \varepsilon$. 


\section{Proof of the existence theorem}

3.1. A minimax principle. Our main tool for proving Theorem 1.6 will be an abstract minimax principle due to El Amrouss and Moussaoui [9].

Theorem 3.1. Let $\Phi$ be a $C^{1}$ functional on $X$ satisfying condition $(C)$, let $Q$ be a closed connected subset of $X$ such that $\partial Q \cap \partial(-Q) \neq \emptyset$, and let $\beta \in \mathbb{R}$. Assume that

(1) for every $K \in \mathscr{C}_{2}$ there exists $v_{K} \in K$ such that $\Phi\left(v_{K}\right) \geq \beta$ and $\Phi\left(-v_{K}\right)$ $\geq \beta$,

(2) $a=\sup _{\partial Q} \Phi<\beta$,

(3) $\sup _{Q} \Phi<\infty$.

Then $\Phi$ has a critical value $c \geq \beta$ given by

$$
c=\inf _{h \in \Gamma} \sup _{x \in Q} \Phi(h(x)),
$$

where $\Gamma=\{h \in C(X, X): h(x)=x$ for every $x \in \partial Q\}$.

\subsection{Compactness conditions}

Lemma 3.2. Suppose that F satisfies (1.6), (1.8), and (1.22). Then the functional $\Phi$, given by (1.2), satisfies the Cerami condition.

Proof. In a similar way to [9, Lemma 3.1], we see that the first condition in Definition 1.1 holds.

We will prove that the second condition in Definition 1.1 holds, in the case $L(x, s, t) \rightarrow-\infty$ as $\|(s, t)\| \rightarrow \infty$ (the case $L(x, s, t) \rightarrow+\infty$ is similar). To do that, assume by contradiction that there exists a sequence $\left(u_{n}, v_{n}\right)_{n \in \mathbb{N}} \subset W$ such that

$$
\Phi\left(u_{n}, v_{n}\right) \longrightarrow c, \quad \varepsilon_{n}=\left\|\Phi^{\prime}\left(u_{n}, v_{n}\right)\right\|\left\|\left(u_{n}, v_{n}\right)\right\| \longrightarrow 0, \quad\left\|\left(u_{n}, v_{n}\right)\right\| \longrightarrow \infty
$$

Therefore,

$$
\left|\frac{1}{p}\left\langle\Phi_{u}\left(u_{n}, v_{n}\right), u_{n}\right\rangle+\frac{1}{q}\left\langle\Phi_{v}\left(u_{n}, v_{n}\right), v_{n}\right\rangle-\Phi\left(u_{n}, v_{n}\right)\right| \longrightarrow c
$$

or equivalently

$$
\lim _{n \rightarrow \infty}\left|\int_{\Omega} \frac{1}{p} F_{u}\left(x, u_{n}, v_{n}\right) u_{n}+\frac{1}{q} F_{v}\left(x, u_{n}, v_{n}\right) v_{n}-F\left(x, u_{n}, v_{n}\right)\right|=c .
$$

We define

$$
z_{n}=\alpha_{n}^{1 / p} u_{n}, \quad w_{n}=\alpha_{n}^{1 / q} v_{n}
$$

where

$$
\alpha_{n}=\frac{1}{\mathscr{A}_{0}\left(u_{n}, v_{n}\right)} \longrightarrow 0
$$


164 Quasilinear elliptic systems of resonant type

with $\mathscr{A}_{0}$ given by definition (2.8). We have that $\mathscr{A}_{0}\left(z_{n}, w_{n}\right)=1$ so $\left(z_{n}, w_{n}\right)$ is bounded in $W$. After passing to a subsequence, we may assume that

$$
\begin{array}{cl}
z_{n} \rightarrow z & \text { in } W^{1, p}(\Omega), \\
w_{n} \longrightarrow w & \text { in } W^{1, q}(\Omega), \\
z_{n} \longrightarrow z & \text { in } L^{p}(\Omega), \text { a.e. in } \Omega, \\
w_{n} \longrightarrow w & \text { in } L^{q}(\Omega) \text {, a.e. in } \Omega .
\end{array}
$$

Now we show that $(z, w) \neq(0,0)$

$$
\frac{\Phi\left(u_{n}, v_{n}\right)}{\mathscr{A}_{0}\left(u_{n}, v_{n}\right)}=1-\frac{\int_{\Omega} F\left(x, u_{n}, v_{n}\right)}{\mathscr{A}_{0}\left(u_{n}, v_{n}\right)} .
$$

From (1.22), we get that for any $\varepsilon>0$, there exists $C_{\varepsilon}>0$ such that

$$
F(x, s, t) \leq\left(a_{2}(x)+\varepsilon\right) G_{2}(s, t)+C_{\varepsilon} .
$$

As a consequence

$$
\int_{\Omega} F\left(x, u_{n}, v_{n}\right) \leq \int_{\Omega}\left(a_{2}(x)+\varepsilon\right) G_{2}\left(u_{n}, v_{n}\right)+C_{\varepsilon}|\Omega|,
$$

then

$$
\frac{\int_{\Omega} F\left(x, u_{n}, v_{n}\right)}{\mathscr{A}_{0}\left(u_{n}, v_{n}\right)} \leq \alpha_{n} \int_{\Omega}\left(a_{2}(x)+\varepsilon\right) G_{2}\left(u_{n}, v_{n}\right)+C_{\varepsilon}|\Omega| \alpha_{n} .
$$

Since

$$
\alpha_{n} \int_{\Omega}\left(a_{2}(x)+\varepsilon\right) G_{2}\left(u_{n}, v_{n}\right)=\int_{\Omega}\left(a_{2}(x)+\varepsilon\right) G_{2}\left(z_{n}, w_{n}\right)
$$

in the limit we get

$$
0 \geq 1-\int_{\Omega}\left(a_{2}(x)+\varepsilon\right) G_{2}(z, w)
$$

and we conclude that $G_{2}(z, w) \not \equiv 0$.

Let

$$
L(x, s, t)=\frac{1}{p} F_{s}(x, s, t) s+\frac{1}{q} F_{t}(x, s, t) t-F(x, s, t) .
$$

By (1.8) (and since $L$ is continuous), $L(x, s, t) \leq-M$. It follows that

$$
\int_{\Omega} L\left(x, u_{n}, v_{n}\right) \leq \int_{\left\{G_{2}(z, w) \neq 0\right\}} L\left(x, u_{n}, v_{n}\right)+M\left|\left\{x: G_{2}(z(x), w(x))=0\right\}\right| .
$$

Note that

$$
\alpha_{n} G_{2}\left(u_{n}, v_{n}\right) \longrightarrow G_{2}(z, w)
$$


So in the set $\left\{x: G_{2}(z(x), w(x)) \neq 0\right\}, G_{2}\left(u_{n}, v_{n}\right) \rightarrow+\infty$, and then by (1.11), we have that $u_{n}(x), v_{n}(x) \rightarrow \infty$. It follows that $L\left(u_{n}, v_{n}\right) \rightarrow-\infty$ by condition (1.8). Hence the first integral tends to $-\infty$ by Fatou lemma, and we get

$$
\lim _{n \rightarrow \infty} \int_{\Omega} L\left(x, u_{n}, v_{n}\right)=-\infty .
$$

This contradicts (3.4), and the proof is completed.

3.3. Geometric conditions. In this section we show that the functional $\Phi$ satisfies the geometric conditions of Theorem 3.1.

Lemma 3.3. Let $F$ satisfy the assumptions of Theorem 1.6. Then the functional $\Phi$, given by (1.2), satisfies

(1) there exists $(\varphi, \psi) \in W$ such that $\Phi\left(c^{1 / p} \varphi, c^{1 / q} \psi\right) \rightarrow-\infty$ as $c \rightarrow+\infty$;

(2) for every $K \in \mathscr{C}_{2}$ there exists $\left(u_{K}, v_{K}\right) \in K$ and $\beta \in \mathbb{R}$ such that $\Phi\left(u_{K}, v_{K}\right) \geq$ $\beta$ and $\Phi\left(-u_{K},-v_{K}\right) \geq \beta$.

Proof. (1) As $\lambda_{1}\left(a, G_{1}\right)<0$, we may choose $\varepsilon>0$ such that $\lambda_{1}\left(a_{1}-\varepsilon, G_{1}\right)<0$. Let $(\varphi, \psi)$ be the first eigenfunction for the problem

$$
\begin{aligned}
-\Delta_{p} u-\left(a_{1}(x)-\varepsilon\right) G_{1 u}(u, v) & =\lambda|u|^{p-2} u & & \text { in } \Omega, \\
-\Delta_{q} v-\left(a_{1}(x)-\varepsilon\right) G_{1 v}(u, v) & =\lambda|v|^{q-2} v & & \text { in } \Omega, \\
u=v & =0 & & \text { in } \partial \Omega,
\end{aligned}
$$

normalized by

$$
\frac{1}{p} \int_{\Omega}|\varphi|^{p}+\frac{1}{q} \int_{\Omega}|\psi|^{q}=1
$$

Then, using (1.13), we get

$$
\frac{1}{p} \int_{\Omega}|\nabla \varphi|^{p}+\frac{1}{q} \int_{\Omega}|\nabla \psi|^{q}-\int_{\Omega}\left(a_{1}(x)-\varepsilon\right) G_{1}(u, v)=\lambda_{1}\left(a_{1}-\varepsilon, G_{1}\right) .
$$

By (1.22), we have

$$
F(x, s, t) \geq\left(a_{1}(x)-\varepsilon\right) G_{1}(s, t)-C_{\varepsilon} .
$$

It follows that

$$
\begin{aligned}
\Phi\left(c^{1 / p} \varphi, c^{1 / q} \psi\right) \leq & c\left(\frac{1}{p} \int_{\Omega}|\nabla \varphi|^{p}+\frac{1}{q} \int_{\Omega}|\nabla \psi|^{q}\right. \\
& \left.\quad-\int_{\Omega}\left(a_{1}(x)-\varepsilon\right) G_{1}(\varphi, \psi)\right)+C_{\varepsilon}|\Omega| \\
\leq & c \lambda_{1}\left(a_{1}-\varepsilon, G_{1}\right)+C_{\varepsilon}|\Omega|,
\end{aligned}
$$

and so $\Phi\left(c^{1 / p} \varphi, c^{1 / q} \psi\right) \rightarrow-\infty$ as $c \rightarrow+\infty$. 
(2) Since $\lambda_{2}\left(a_{2}, G_{2}\right)>0$, we may choose $\varepsilon>0$ such that $\lambda_{2}\left(a_{2}+\varepsilon, G_{2}\right)>0$. Given $K \in \mathscr{C}_{2}$ and this $\varepsilon>0$, we claim that there exists $\left(u_{K}, v_{K}\right) \in K$ verifying

$$
\begin{aligned}
& \lambda_{2}\left(a_{2}+\varepsilon, G_{2}\right)\left(\frac{1}{p} \int_{\Omega}\left|u_{K}\right|^{p}+\frac{1}{q} \int_{\Omega}\left|v_{K}\right|^{q}\right) \\
& \leq \frac{1}{p} \int_{\Omega}\left|\nabla u_{K}\right|^{p}+\frac{1}{q} \int_{\Omega}\left|\nabla v_{K}\right|^{q}-\int_{\Omega}\left(a_{2}(x)+\varepsilon\right) G_{2}\left(u_{K}, v_{K}\right) .
\end{aligned}
$$

By (1.22), we have

$$
F(x, s, t) \leq\left(a_{2}(x)+\varepsilon\right) G_{2}(s, t)+C_{\varepsilon}
$$

It follows that

$$
\begin{aligned}
\Phi\left(u_{K}, v_{K}\right) \geq & \frac{1}{p} \int_{\Omega}\left|\nabla u_{K}\right|^{p}+\frac{1}{q} \int_{\Omega}\left|\nabla v_{K}\right|^{q} \\
& -\int_{\Omega}\left(a_{2}(x)+\varepsilon\right) G_{2}\left(u_{K}, v_{K}\right)-C_{\varepsilon}|\Omega| \\
\geq & \lambda_{2}\left(a_{2}+\varepsilon, G_{2}\right)\left(\frac{1}{p} \int_{\Omega}\left|u_{K}\right|^{p}+\frac{1}{q} \int_{\Omega}\left|v_{K}\right|^{q}\right)-C_{\varepsilon}|\Omega| \\
\geq & -C_{\varepsilon}|\Omega|=\beta .
\end{aligned}
$$

Similarly,

$$
\Phi\left(-u_{K},-v_{K}\right) \geq-C_{\varepsilon}|\Omega|=\beta
$$

3.4. Proof of Theorem 1.6. We apply Theorem 3.1. We take

$$
Q=\left\{\left(|c|^{1 / p-1} c \varphi,|c|^{1 / q-1} c \psi\right),-R \leq c \leq R\right\},
$$

where $(\varphi, \psi)$ is given by Lemma 3.3. $Q$ is closed and compact (it is the image of $[-R, R]$ under a continuous mapping). Also $\partial Q=\partial(-Q)=\left\{\left( \pm R^{1 / p} \varphi, \pm R^{1 / q} \psi\right)\right\} \neq$ $\emptyset$. By Lemma 3.3 if we choose $R$ big enough, we have

$$
\sup _{\partial Q} \Phi<\beta
$$

Also $\sup _{Q} \Phi<+\infty$ since $Q$ is compact and $\Phi$ is continuous. The functional $\Phi$ verifies condition $(C)$ by Lemma 3.2. Then all the conditions of Theorem 3.1 are fulfilled and the proof is completed.

\section{Acknowledgment}

The authors thank specially Prof. J. P. Gossez and the referee for their careful reading of the manuscript and their fruitful suggestions and remarks. 


\section{References}

[1] H. Amann, Lusternik-Schnirelman theory and non-linear eigenvalue problems, Math. Ann. 199 (1972), 55-72.

[2] P. Bartolo, V. Benci, and D. Fortunato, Abstract critical point theorems and applications to some nonlinear problems with "strong" resonance at infinity, Nonlinear Anal. 7 (1983), no. 9, 981-1012.

[3] J. M. Bezerra do Ó, Solutions to perturbed eigenvalue problems of the p-Laplacian in $\mathbb{R}^{n}$, Electron. J. Differential Equations 1997 (1997), 1-15.

[4] L. Boccardo and D. G. de Figueiredo, Some remarks on a system of quasilinear elliptic equations, preprint, 1997, http://www.ime.unicamp.br/rel_pesq/ 1997/rp51-97.html.

[5] G. Cerami, Un criterio di esistenza per i punti critici su varieta' illimitate [An existence criterion for the critical points on unbounded manifolds], Istit. Lombardo Accad. Sci. Lett. Rend. A 112 (1978), no. 2, 332-336 (Italian).

[6] M. M. Day, Some more uniformly convex spaces, Bull. Amer. Math. Soc. 47 (1941), 504-507.

[7] P. de Nápoli and M. C. Mariani, Three solutions for quasilinear equations in $\mathbb{R}^{n}$ near resonance, Proceedings of the USA-Chile Workshop on Nonlinear Analysis (Viña del Mar-Valparaiso, 2000), Southwest Texas State Univ., Texas, 2001, pp. 131-140.

[8] G. Dinca, P. Jebelean, and J. Mawhin, Variational and Topological Methods for Dirichlet Problems with p-Laplacian, Recherches de mathématique, Inst. de Math. Pure et Apliquée, Univ. Cath. de Louvain, 1998.

[9] A. R. El Amrouss and M. Moussaoui, Minimax principles for critical-point theory in applications to quasilinear boundary-value problems, Electron. J. Differential Equations 2000 (2000), no. 18, 1-9.

[10] J. Fleckinger, R. F. Manásevich, N. M. Stavrakakis, and F. de Thélin, Principal eigenvalues for some quasilinear elliptic equations on $\mathbf{R}^{N}$, Adv. Differential Equations 2 (1997), no. 6, 981-1003.

[11] J.-P. Gossez, Some remarks on the antimaximum principle, Rev. Un. Mat. Argentina 41 (1998), no. 1, 79-83.

Pablo L. de Nápoli: Departamento de Matemática, Facultad de Ciencias Exactas y Naturales, Universidad de Buenos Aires, Ciudad Universitaria, Pabellón I, (1428) Buenos Aires, Argentina

E-mail address: pdenapo@dm.uba.ar

M. Cristina Mariani: Departamento de Matemática, Facultad de Ciencias Exactas y Naturales, Universidad de Buenos Aires, Ciudad Universitaria, Pabellón I, (1428) Buenos Aires, Argentina

E-mail address: mcmarian@dm.uba.ar 


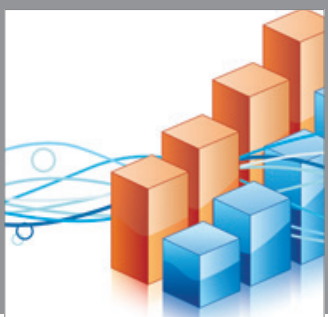

Advances in

Operations Research

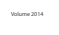

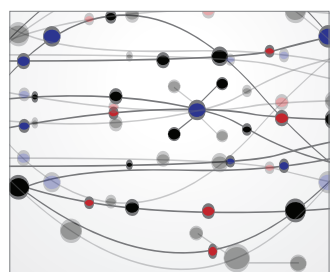

\section{The Scientific} World Journal
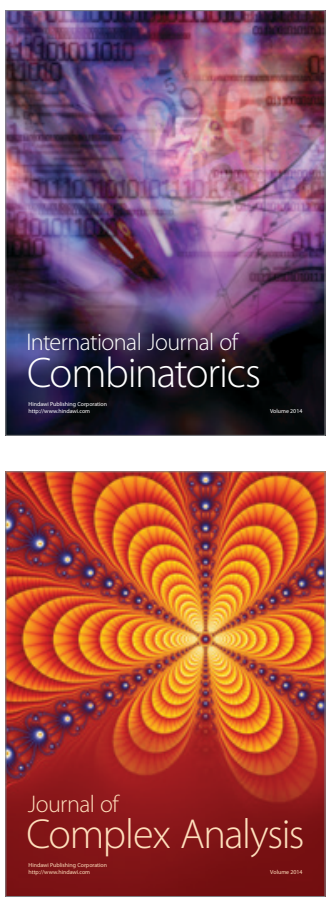

International Journal of

Mathematics and

Mathematical

Sciences
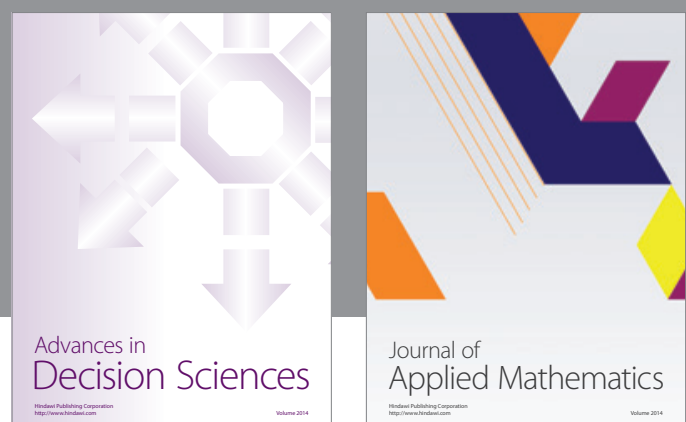

Journal of

Applied Mathematics
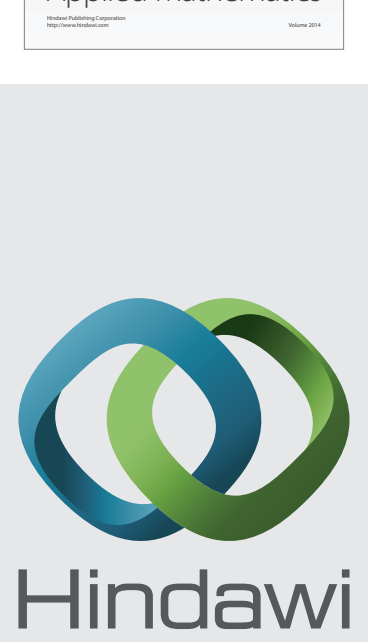

Submit your manuscripts at http://www.hindawi.com
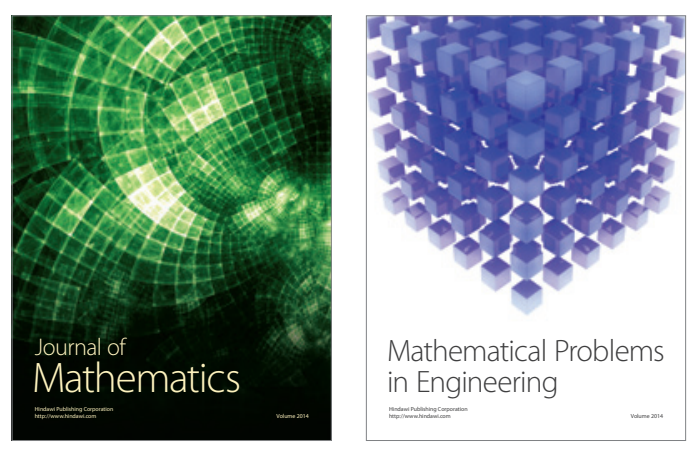

Mathematical Problems in Engineering
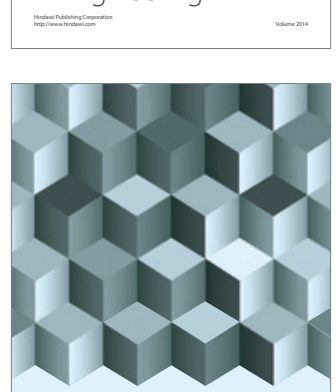

Journal of

Function Spaces
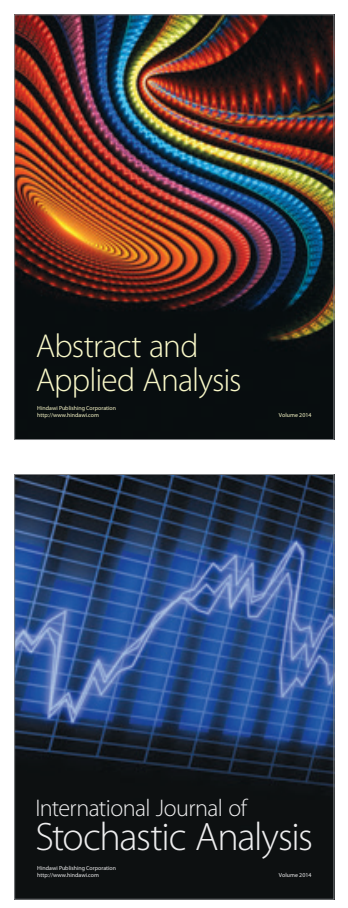

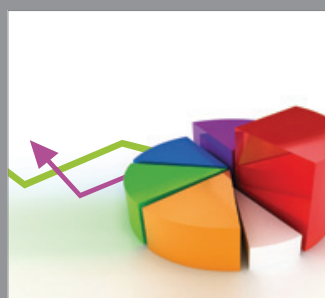

ournal of

Probability and Statistics

Promensencen
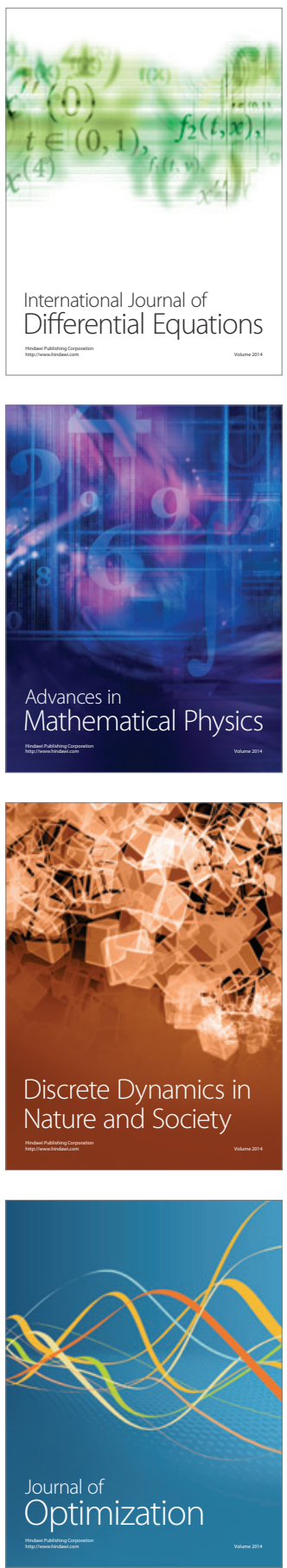